\title{
Intelligent Fault Diagnosis of 3D Printers based on Reservoir Computing
}

\author{
Xiang Duan ${ }^{\mathrm{a}, \mathrm{b}}$, Jianyu Long ${ }^{\mathrm{a}}$, Chuan $\mathrm{Li}^{\mathrm{a}}$, Diego Cabrera ${ }^{\mathrm{c}}$, and Shaohui Zhang ${ }^{\mathrm{a},{ }^{*}}$ \\ ${ }^{a}$ School of Mechanical Engineering, Dongguan University of Technology, Dongguan, 523808, China \\ ${ }^{b}$ College of Mechatronics and Control Engineering, Shenzhen University, Shenzhen, 518060, China \\ ${ }^{c}$ GIDTEC, Universidad Politécnica Salesiana, Ecuador
}

\begin{abstract}
Fault diagnosis is important for the working conditions of 3D printers, because the failure of 3D printers will have a great impact on the quality of printed products and result in unqualified printing. In this paper, the reservoir computing (RC) method and the data collected by the attitude sensor are analyzed to obtain the health status of a 3D printer. Considering the economics and viability of fault diagnosis, a low-cost attitude sensor is installed on the moving platform of the 3D printer to collect tri-axial angular velocity, tri-axial acceleration, and tri-axial magnetic field strength signals. Then, the collected data is divided into training data and test data. The training data is used to establish the optimization parameter of the RC model to improve its performance, and the test data is used to identify the failure patterns using the model. Finally, compared with the SAE and SVM intelligent diagnosis techniques, the RC method achieves the best fault recognition accuracy, which further proves its superiority.
\end{abstract}

Keywords: 3D printer; pattern recognition; fault diagnosis; reservoir computing

(Submitted on October 3, 2019; Revised on November 5, 2019; Accepted on November 17, 2019)

(C) 2019 Totem Publisher, Inc. All rights reserved.

\section{Introduction}

The topic of 3D printing continues to be popular today. 3D printing is also known as additive manufacturing, which is based on the technical principle of fusion deposition modeling (FDM). It forms a layer of deposited material by extrusion of the molten polymer filaments [1] and the movement of the hot nozzle according to the geometrical position. Compared with traditional mold preparation methods [2], 3D printing has the advantages of saving materials, shortening the product development cycle [3], and creating almost any geometric shape, which is why 3D printing is still popular today. Additive manufacturing is widely used in various industries, such as rapid modeling [4], materials [5], and biomedical engineering [6]. The failure of a 3D printer will result in poor quality of the printed product, which will eventually lead to downtime repair and economic losses. Therefore, it is necessary to diagnose 3D printers to control the quality of printed products and reduce losses.

It is well known that during normal operation of a machine, friction and wear between components can occur, which affects the operational state of the machine. At this time, we can analyze the health of the machine from the collected data and some methods of fault diagnosis [7], such as analyzing the machine's vibration [8], electricity [9], thermal [10], and acoustic signals [11] to assess the condition of the machine. There are many fault detection and diagnostic methods that are widely used in industries [12]. Park et al. [13] proposed a method of positive energy residual, which analyzes gear fault detection by extracting the vibration signal of the planetary gear under variable speed conditions. Yang et al. [14] studied the problem of extracting fault features caused by noise interference and proposed a new method based on variable-mode decomposition and phase-space parallel factor analysis. The results showed that the method can be applied to weak fault signals of rolling bearings. Due to different transmission mechanisms, the above proposed methods may not be applicable to $3 \mathrm{D}$ printers. Therefore, the purpose of this paper is to design an effective fault diagnosis method for 3D printers.

With the development of science and technology, artificial intelligence is being widely used in various fields, and

\footnotetext{
* Corresponding author.

E-mail address: shaohui1985@hotmail.com
} 
machine learning is a relatively popular research direction. Recently, with the wide application of machine learning in computer vision and speech recognition, machine learning has begun to be applied in the field of fault diagnosis [15]. Guo et al. [16] used extreme learning machine (ELM) in the fault diagnosis of asynchronous motor drive systems. The research results showed that the method has high accuracy for position detection of fault sensors and can identify specific fault information, such as offset, stuck, and noise. Li et al. [17] combined the stacked automatic encoder (SAE) and long-term short-term memory (LSTM) neural networks for the fault diagnosis analysis of mechanical equipment. The experimental results showed that the method can achieve a 99\% accurate diagnosis rate. Support vector machine (SVM) [18] is a machine learning algorithm. In the field of fault diagnosis, SVM is one of the most common methods of discriminating. It is a supervised learning model commonly used for pattern recognition, classification, and regression analysis. Santos et al. [19] proposed a method of SVM classifier with a multi-sensory system for the fault detection of wind turbines. Li et al. [20] proposed a method of multimodal deep support vector classification for the fault diagnosis of gearboxes. However, SVM does not provide a general solution to nonlinear problems.

Neural networks have good nonlinear mapping capabilities, so they have attracted wide attention in the field of machine learning. Among the series of techniques for deep learning, there is an important model called artificial neural network (ANN) [21], which is a complex network structure formed by a large number of processing elements (called neurons) connected to each other. A neural network is a mathematical model that simulates neuronal activity, and it is an information processing system similar to the human brain. One popular ANN is the recurrent neural network (RNN) [22]. It is a neural network that creates directional connections into neurons of the same layer. It has the advantage of being able to process input sequences of any timing. However, in the practical application of neural networks, there are still many problems. For example, the recurrent neural network has a large amount of computation and a slow convergence rate, and the number of hidden neurons is difficult to determine.

In addition, Jaeger proposed the echo state network (ESN) in 2001 [23], and Mass proposed the liquid state machine (LSM) in 2002 [24]. Although the research methods of these models are different, they are both improvements to the traditional recurrent neural network algorithm. Verstraeten et al. [25] found that ESN and LSM have the same properties, so they are collectively referred to as reservoir computing (RC). As a new machine learning method, RC is widely used in time series prediction, pattern classification, nonlinear system modeling, identification and control, etc. with its unique dynamic RC structure and simple learning algorithm; it can also be used in the field of fault diagnosis. Cabrera et al. [26] used RC and variation reasoning to address the fault detection of rotating machinery as a one-class learning problem. Zheng et al. [27] first attempted to apply RC to the field of fuel cells. Therefore, this paper attempts to apply RC to the field of fault diagnosis of $3 \mathrm{D}$ printers.

The analyzed delta 3D printer is a typical parallel-structure printer and has a very complicated transmission structure, so it is prone to failure. He et al. [28] proposed a method of attitude sensor monitoring and diagnosed the 3D printer through the SVM model, obtaining a diagnostic accuracy of $94.44 \%$. However, they used expensive attitude sensors in the experiment, which have strong anti-interference ability. Thus, the collected information was orderly, and good diagnostic accuracy could be obtained by using a simple SVM algorithm. Our philosophy is to reduce the cost of the experiment by collecting data through low-cost sensors and then processing the data through advanced algorithms. In order to consider the economics and feasibility of fault diagnosis, this paper uses a low-cost attitude sensor to collect tri-axial angular velocity, tri-axial acceleration, and tri-axial magnetic field strength signals. We aim to solve the problem that there is a large amount of interference information in the data collected by a low-cost sensor. Firstly, we establish an RC model by tuning the model parameters of the experimental data, and then we obtain the improved model by optimizing the parameters of the RC model. The experimental results show that the model has good fault recognition accuracy. Therefore, this paper proposes a method of monitoring with attitude sensors, which combines the method of RC and establishes an RC model for the fault diagnosis of $3 \mathrm{D}$ printers.

The remainder of this paper is organized as follows. The principles and experimental steps for RC are presented in Section 2. In Section 3, the types of faults in 3D printers are described. Then, it is recommended to use the real-time monitoring method of the attitude sensor for analysis. In Section 4, the results and discussion of the experiment are analyzed. Finally, Section 5 gives the conclusion.

\section{Methodology}

This section describes the system approach for RC. Its working schematics and calculation formulas are introduced step by step in the following sections. The entire design process of the experimental steps is given in the last section. 


\subsection{Reservoir Computing (RC)}

As shown in Figure 1, the RC architecture consists of three different sets of neurons: the input layer, the reservoir layer, and the output layer. Among them, the sample data matrix is used as the input layer, the nonlinear time-expanded map of the input signal is used as the reservoir, and the fault pattern recognition accuracy of the 3D printer is used as the output layer. It is assumed that the number of neurons input to the reservoir layer is $\mathrm{A}$, the number of neurons in the output layer is $\mathrm{C}$, and there are B units in the reservoir. When the time is $t$, the input of the system is $\mathbf{x}=\left\{x_{1}(t), \cdots, x_{A}(t)\right\}$, the result of the internal state of the reservoir is $\mathbf{u}=\left\{u_{1}(t), \cdots, u_{B}(t)\right\}$, the result of the system output is $\mathbf{y}=\left\{y_{1}(t), \cdots y_{C}(t)\right\}$, and the state update equation for managing the network is

$$
\begin{gathered}
\mathbf{u}(t)=f\left(\mathbf{W}_{1} \mathbf{x}(t)+\mathbf{W}_{2} \mathbf{u}(t-1)\right) \\
\mathbf{y}(t)=\mathbf{W}_{3}[\mathbf{u}(t) ; \mathbf{x}(t)]
\end{gathered}
$$

Where $\mathbf{W}_{1}$ is a matrix of $B \times A$, which is the connection weight matrix between the feature reinforcement matrix and the internal unit of the reservoir. $\mathbf{W}_{2}$ is a matrix of $B \times B$, which is the connection weight of the internal unit. $\mathbf{W}_{3}$ is a matrix of $C \times(A+B)$, which is the connection weight of the internal unit and the output. In Equation (1), $f(\cdot)$ is the activation function. The vector $[\mathbf{u}(t) ; \mathbf{x}(t)]$ in Equation (2) is the extended state vector connecting the reservoir and the input state.

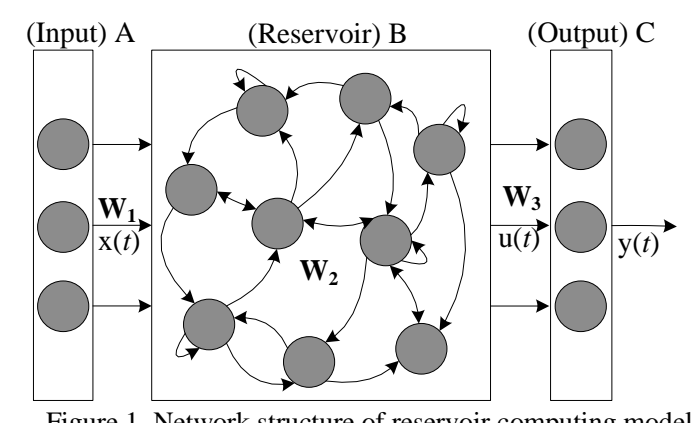

In the RC system, the weights $\mathbf{W}_{1}, \mathbf{W}_{2}$, and $\mathbf{W}_{3}$ are independently determined and optimized. The sparse matrixes for $\mathbf{W}_{1}$ and $\mathbf{W}_{2}$ have the sample initialization distribution of the $\mathbf{W}_{3}$. When the values of $\mathbf{W}_{1}$ and $\mathbf{W}_{2}$ are determined (i.e., a stable reservoir is obtained), $\mathbf{W}_{3}$ can be optimized by solving the optimization problem. Therefore, the output weight $\mathbf{W}_{3}$ is

$$
\begin{gathered}
\mathbf{W}_{3}=\underset{\mathbf{W}_{3}}{\arg \min } \operatorname{MSE}\left(\mathbf{y}^{\text {expect }}, \mathbf{y}\right) \\
\operatorname{MSE}\left(\mathbf{y}^{\text {expect }}, \mathbf{y}\right)=\frac{1}{Q} \frac{1}{T} \sum_{i=1}^{Q} \sum_{t=1}^{T}\left[\mathbf{y}_{i}^{\text {expect }}(t)-\mathbf{y}_{i}(t)\right]^{2}
\end{gathered}
$$

\subsection{Overview of Attitude Monitoring based on RC Modeling}

Based on the above analysis, the experimental steps are shown in Figure 2.

(1) Install an attitude sensor on the 3D printer mobile platform.

(2) Collect data for different fault patterns.

(3) All channel data on the sensor are used to generate training and test samples with given labels.

(4) Train the RC model to obtain the optimal RC model. 
(5) Input the test samples into the optimized RC model.

(6) Output the health condition of the 3D printer.

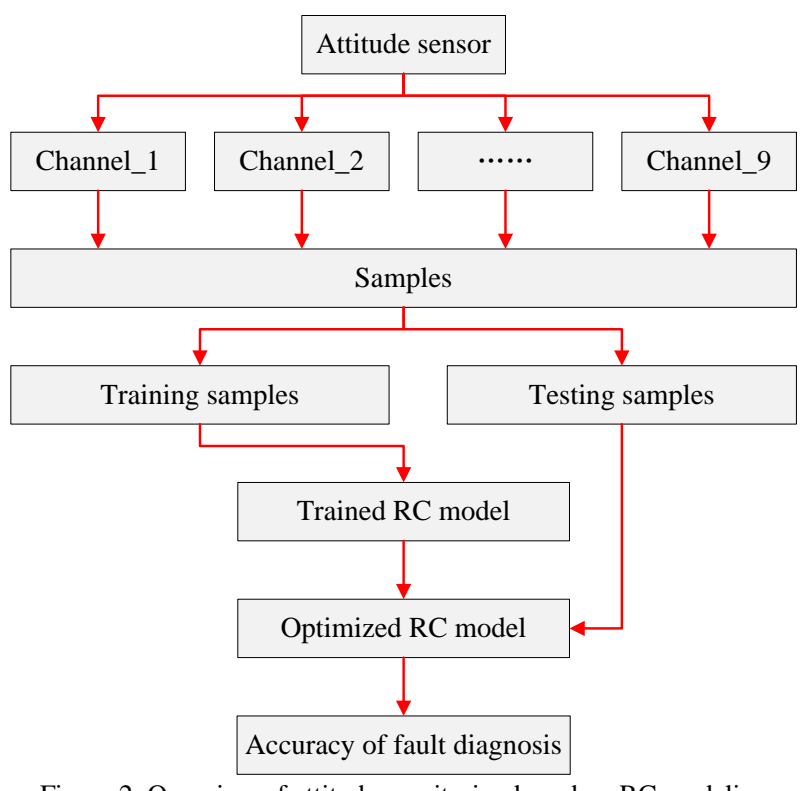

Figure 2. Overview of attitude monitoring based on RC modeling

\section{Experiments}

In order to verify the feasibility of the reservoir computing, an experimental setup is established as shown in Figure 3 . The experiment is set up with a 3D printer, and an attitude sensor is connected to the computer. This sensor can measure tri-axial acceleration, tri-axial magnetic fields, and tri-axial angular velocity. The angular velocity, attitude measurement, and acceleration stability of the sensor are $0.05 \%, 0.01^{\circ}$, and $0.01 \mathrm{~g}$, respectively. This particular sensor was selected because it is very inexpensive (less than $\$ 20$ per piece).

The experimental 3D printer is shown in Figure 3, and it consists of three sliders, three vertical rails, three parallel arms, several synchronous belts, and a moving platform. The parallel arm, slider, and moving platform are connected by a ball joint (also called a joint bearing), as shown in Figure 4(a). The movement of the slider can be achieved by a motor drive connected by a synchronous belt. According to the relevant structural analysis of the 3D printer, it is found that the wear and clearance of the joint bearing may cause the moving and building platform to be non-parallel and affect the quality of the printed product, which we refer to as printer failure.

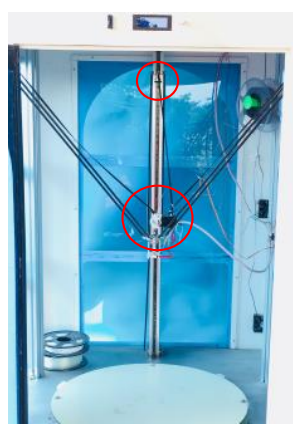

(a)

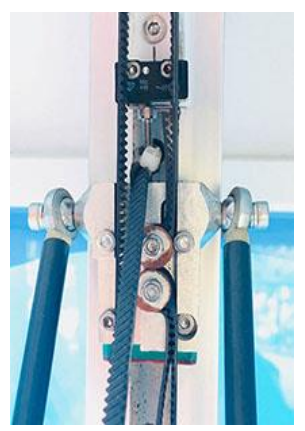

(b)

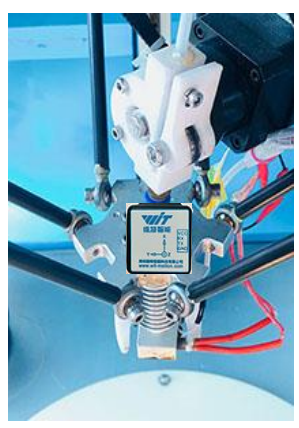

(c)

Figure 3. Experimental configurations for the fault diagnosis: (a) 3D printer, (b) slider, and (c) moving platform

In our scheme, the Repetier-Host (slicing software) provides the G code for the 3D printer to run the printer and then collects the data from the attitude sensor on the moving platform. The calibration attitude sensor can adjust the sampling frequency by observing real-time data. In this work, the sampling frequency is set to $100 \mathrm{~Hz}$. 


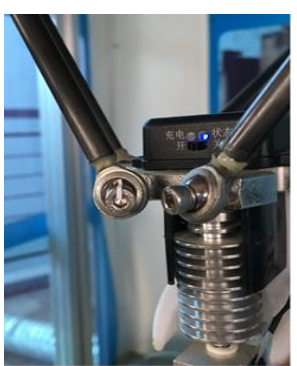

(a)

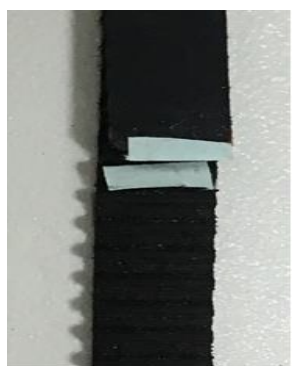

(b)

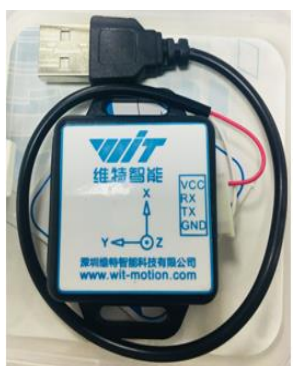

(c)

Figure 4. The fault location and sensor in the experiment: (a) joint bearing, (b) synchronous belt, and (c) attitude sensor

The wear of different joint bearings and synchronous belts are set as a wrong pattern in Figure 4 . There are 12 joint bearings and three synchronous belts in the 3D printer. In general, we have set 16 failure patterns in the experiment, as shown in Table 1. Because the slider, the moving platform, and the joint bearing are connected by threads in the printer, the screw spacing of each joint bearing is $0.70 \mathrm{~mm}$, so we loosen the screw of each joints bearing by 2.00 cycles to simulate the fault. Therefore, we can obtain 12 joint bearing faults, labeled as A, B, C, $\cdots$, and L. Since the length of the synchronous belt changes due to elastic deformation after a long period of use (i.e., printer failure), we loosen the length of 1.00 tooth for each of the synchronous belts as a simulation of the failure. Thus, we can collect three simulated synchronous belt faults labeled as M, N, and O. Finally, the experimental result under normal conditions is labeled as P.

Table 1. Condition patterns set in the experiments

\begin{tabular}{cccccc}
\hline $\begin{array}{c}\text { Failure } \\
\text { number }\end{array}$ & Fault location & $\begin{array}{c}\text { Degree of } \\
\text { failure }\end{array}$ & $\begin{array}{c}\text { Failure } \\
\text { number }\end{array}$ & Fault location & $\begin{array}{c}\text { Degree of } \\
\text { failure }\end{array}$ \\
\hline A & joint bearing a & 2.00 laps & I & joint bearing i & 2.00 laps \\
B & joint bearing b & 2.00 laps & J & joint bearing j & 2.00 laps \\
C & joint bearing c & 2.00 laps & K & joint bearing k & 2.00 laps \\
D & joint bearing d & 2.00 laps & L & joint bearing 1 & 2.00 laps \\
E & joint bearing e & 2.00 laps & M & synchronous belt a & 1.00 tooth \\
F & joint bearing f & 2.00 laps & N & synchronous belt b & 1.00 tooth \\
G & joint bearing g & 2.00 laps & O & synchronous belt c & 1.00 tooth \\
H & joint bearing h & 2.00 laps & P & Normal & Normal \\
\hline
\end{tabular}

During the experiment, a cylindrical shell model with a radius of $75 \mathrm{~mm}$ and a height of $0.3 \mathrm{~mm}$ is established by $3 \mathrm{D}$ modeling software, and then the $\mathrm{G}$ code that generates 20 circles with a specific radius in the slicing software is imported. In each experiment, the motion trajectory of the moving platform is 20 circles (the same radius) on the same layer. Each experiment is repeated three times. The data is collected in real time by an attitude sensor, followed by 60 circular data in each conditional pattern. The data collected in each condition pattern is evenly divided into 150 pieces to obtain 150 samples, so we can obtain a total of $150 \times 16=2,400$ samples. Since the sensor has nine channels and each channel has 620 data acquisition points, we can obtain $2,400 \times 2,790$ samples by fast Fourier transform. All samples are randomly sorted prior to training and testing of the RC model, with $50 \%$ of the samples used for model training and $50 \%$ for testing.

\section{Results and Discussion}

A low-cost attitude sensor installed on the 3D printer moving platform collects attitude data under different fault conditions patterns, as shown in Figure 5, where (a-c) is a tri-axial vibration acceleration signal, (d-f) is a tri-axial magnetic field intensity signal, and (g-i) is a tri-axial angular velocity signal.

In order to analyze the performance of the RC model, we establish three sub-datasets containing only partial conditional patterns, namely 8_classes, 12_classes, and 16_classes, for fault diagnosis modeling. Then, we divide the data of the patterns P, M, N, O, A, B, C, D into the 8_classes datasets, the data of the patterns P, M, N, O, A, B, C, D, E, F, G, H are divided into 12_classes datasets, and the 16_classes datasets contain complete data for all 16 patterns. In the above $50 \%$ of 
the training samples, we randomly select $4 / 5$ as the training samples and $1 / 5$ as the testing sample for the model, and we use the cross-validation method to analyze the parameters of the model.

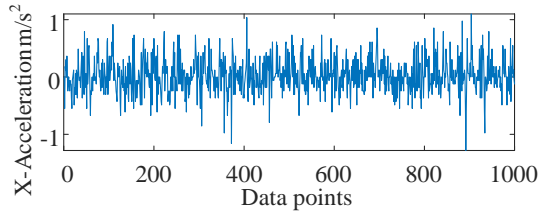

(a)

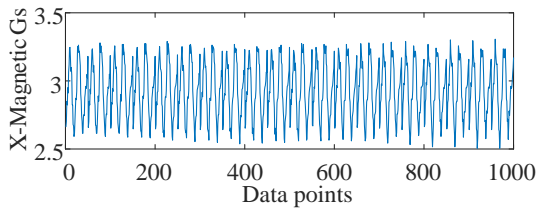

(d)

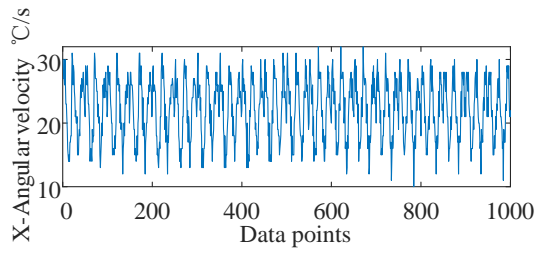

(g)

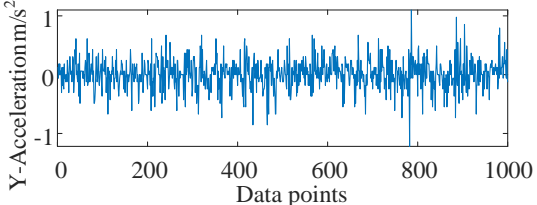

(b)

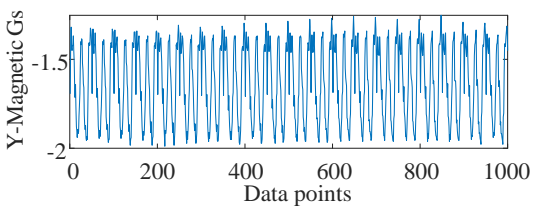

(e)

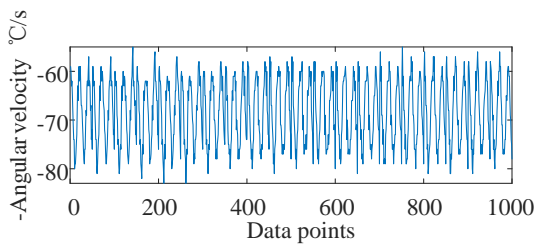

(h)

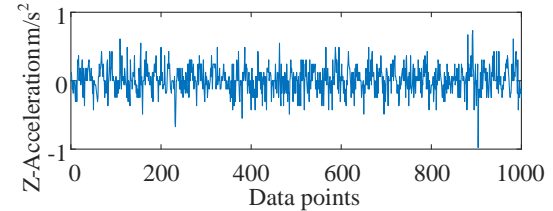

(c)

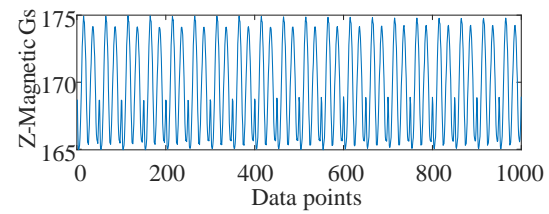

(f)

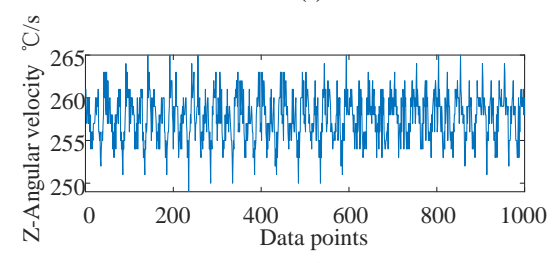

(i)

Figure 5. The data collected from nine channels

Figure 6 shows the parameter analysis of the RC model in sub-datasets of R75. The $X$-axis is the internal connecting weight spectral radius (SR) of the model, the $Y$-axis is the input unit scale (IS) of the model, and the $Z$-axis is the fault recognition rate (accuracy) of the model. From Figure 6(a-c), we can see that when the value of $X$ is in the range of $0-0.1$, and the accuracy of the model is low. However, when the value of $X$ is in the range of $0.1-1.0$, the accuracy of the model is high, showing an improvement effect in this range. Therefore, we further set the parameters of the spectral radius between 0.1 and 1.0 for analysis. In general, we can observe that the accuracy of the model increases with an increase in the spectral radius from these three figures, and good accuracy is mainly concentrated in the lower right of the figure. When the spectral radius of the model is $0.6-1.0$, the accuracy of fault detection is high, so the prediction effect is very good. The marked point in Figure 6(a) is the point where the model has the best result for fault detection, with an accuracy of $94.83 \%$. At this time, the model spectral radius is 1.0, and the input unit scale is 0.1 . In Figure 6(b), the point marked in the figure is a point with the best accuracy for fault detection, and the highest precision is $90.89 \%$. Currently, the model has a spectral radius is 1.0 , and the input unit scale is 0.2 . In Figure 6(c), the highest precision of the model is $83.58 \%$, the spectral radius of the model is 1.0 , and the input unit scale of the model is 0.5 . Therefore, the RC model has the best performance for fault recognition when the spectral radius is 1.0 and the input unit scale is 0.5 .

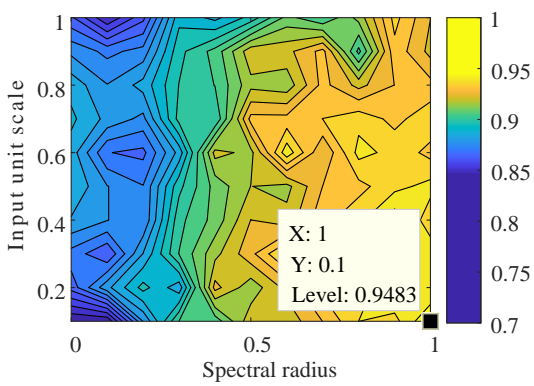

(a)

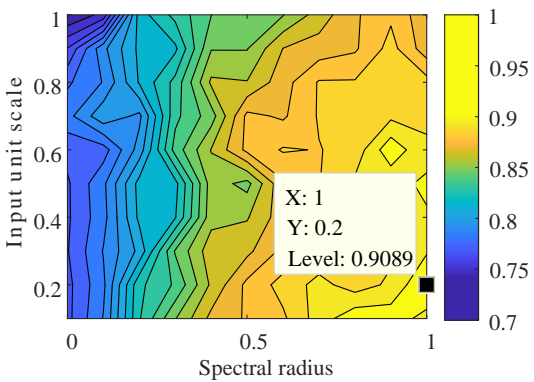

(b)

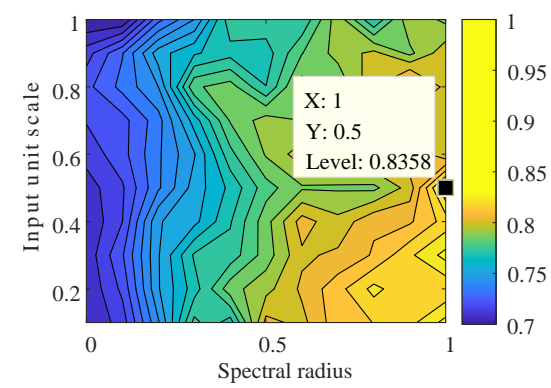

(c)

Figure 6. Parameter analysis of RC models in sub-datasets of R75: (a) 8_classes, (b) 12_classes, and (c) 16_classes datasets

To evaluate the diagnostic performance of the optimized model above, we apply the same experimental data to different methods for comparison. The symmetric structural neural network of sparse auto-encoder (SAE) determines that it can only be used for feature learning, so it is the second comparison method. SVM is one of the most commonly used classifiers, and we choose it as the third comparison method because it has excellent generalization capabilities. During the training process, the input layer node of the model is set to 2,790, the reservoir layer node is set to 100, the output layer node is represented as the number of conditional patterns, and the parameter settings are determined by the above method for hyper parameter 
optimization. The parameters of the training iteration are set to 200, and the parameter of the learning rate is 0.5 . In summary, we obtain the dataset R75 through experiments, and then we train and test the dataset. Finally, the test results are used to diagnose 16 conditional patterns of the 3D printer.

In the above test samples, we respectively input the same samples into the optimized SAE and SVM model. The diagnostic results of the different methods in the R75 sub-datasets are shown in Figure 7. The $X$-axis is the classification of sub-datasets, the $Y$-axis is the recognition accuracy, avg means average diagnostic accuracy, and std means standard deviation. In the 8_classes datasets, we obtain $93.03 \%$ avg and 0.0185 std through the RC model. At the same time, the avg obtained by the SAE model is $75.80 \%$ and the std is 0.0406 , while the avg obtained by the SVM model is $65.37 \%$ and the std is 0.0256 . In the 12_classes datasets, we obtain $90.89 \%$ avg and 0.0067 std through the RC model. Meanwhile, the avg obtained by the SAE model is $69.80 \%$ and the std is 0.0724 , while the avg obtained by the SVM model is $64.40 \%$ and the std is 0.0236 . In the 16_classes datasets, we obtain $82.12 \%$ avg and 0.0163 std through the RC model. The avg obtained by the SAE model is $58.77 \%$ and the std is 0.0525 , while the avg obtained by the SVM model is $50.23 \%$ and the std is 0.0099 , respectively. Therefore, we can conclude the following: first, as the conditional model increases, the accuracy of RC model recognition is less affected, while the recognition accuracy of the SAE and SVM models decreases. This shows that RC performance is more stable than SAE and SVM. Secondly, the recognition accuracy of the RC model is higher in each subdataset than in the SAE and SVM model, which further proves its superiority in the fault diagnosis of 3D printers.

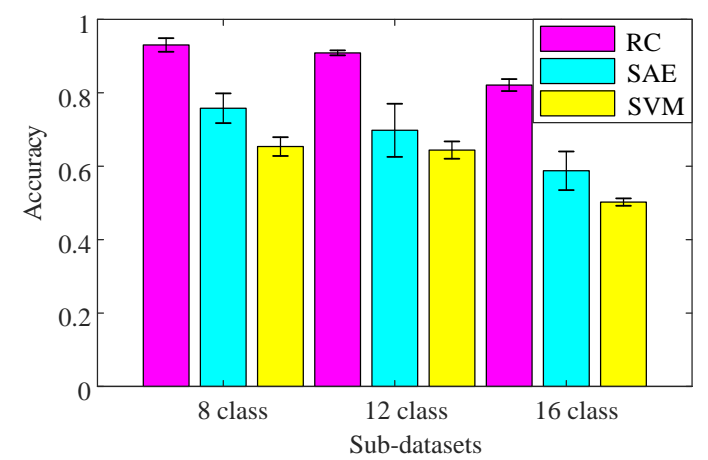

Figure 7. Diagnosis results of different approaches in sub-datasets of R75

\section{Conclusions}

This paper combined the reservoir computing method to analyze the data collected by an attitude sensor and diagnose the faults of 3D printers. The experiment consisted of three steps. First, a low-cost attitude sensor was installed on the moving platform of the 3D printer to collect the attitude data of 16 different failure patterns. Then, 50\% of training label data was used to parameterize the established model to obtain an optimized model, and the data of $50 \%$ of test labels was input into the optimized RC model to identify the failure patterns. Next, an analysis was conducted by collecting actual data from experimental devices such as 3D printers, low cost attitude sensors, and workstations. The parameter analysis of the RC model was mainly studied and compared with the SAE and SVM models. The results showed that RC has the best predictive performance, proving the superiority of $\mathrm{RC}$ in $3 \mathrm{D}$ printer fault diagnosis.

\section{Acknowledgements}

This work is supported in part by the National Natural Science Foundation of China (No. 51775112, 51605406), the Natural Sciences Foundation of Guangdong in China (No. 2017A030313690), and the Research Start-up Funds of DGUT (No. GC300501-12, GC300501-26).

\section{References}

1. F. Calignano, D. Manfredi, E. P. Ambrosio, S. Biamino, M. Lombardi, E. Atzeni, et al., "Overview on Additive Manufacturing Technologies," Proceedings of the IEEE, Vol. 105, No. 4, pp. 593-612, 2017

2. R. E. Rebong, K. T. Stewart, A. Utreja, and A. A. Ghoneima, "Accuracy of Three-Dimensional Dental Resin Models Created by Fused Deposition Modeling, Stereolithography, and Polyjet Prototype Technologies: A Comparative Study," The Angle Orthodontist, Vol. 88, No. 3, pp. 363-369, 2018

3. Z. Sun and D. Liu, "A Systematic Review of Clinical Value of Three-Dimensional Printing in Renal Disease," Quantitative Imaging in Medicine and Surgery, Vol. 8, No. 3, pp. 311, 2018

4. R. L. Truby and J. A. Lewis, "Printing Soft Matter in Three Dimensions," Nature, Vol. 540, No. 7633, pp. 371, 2016 
5. N. Jones, "Science in Three Dimensions: the Print Revolution," Nature News, Vol. 487, No. 7405, pp. 22, 2012

6. M. K. Gupta, F. Meng, B. N. Johnson, Y. L. Kong, L. Tian, Y. W. Yeh, et al., "3D Printed Programmable Release Capsules," Nano Letters, Vol. 15, No. 8, pp. 5321-5329, 2015

7. C. Li, R. V. Sánchez, G. Zurita, M. Cerrada, and D. Cabrera, "Fault Diagnosis for Rotating Machinery using Vibration Measurement Deep Statistical Feature Learning," Sensors, Vol. 16, No. 6, pp. 895, 2016

8. C. Li and M. Liang, "Time-Frequency Signal Analysis for Gearbox Fault Diagnosis using a Generalized Synchrosqueezing Transform," Mechanical Systems and Signal Processing, Vol. 26, pp. 205-217, 2012

9. J. R. Ottewill and M. Orkisz, "Condition Monitoring of Gearboxes using Synchronously Averaged Electric Motor Signals," Mechanical Systems and Signal Processing, Vol. 38, No. 2, pp. 482-498, 2013

10. Y. S. Lu, F. L. Wang, M. X. Jia, and Y. C. Qi, "Centrifugal Compressor Fault Diagnosis based on Qualitative Simulation and Thermal Parameters," Mechanical Systems and Signal Processing, Vol. 81, pp. 259-273, 2016

11. L. X. Gao, F. L. Zai, S. B. Su, H. Q. Wang, P. Chen, and L. M. Liu, "Study and Application of Acoustic Emission Testing in Fault Diagnosis of Low-Speed Heavy-Duty Gears," Sensors, Vol. 11, No. 1, pp. 599-611, 2011

12. S. J. Qin, "Survey on Data-Driven Industrial Process Monitoring and Diagnosis," Annual Reviews in Control, Vol. 36, No. 2, pp. 220-234, 2012

13. J. Park, M. Hamadache, J. M. Ha, Y. Kim, K. Na, and B. D. Youn, "A Positive Energy Residual (PER) based Planetary Gear Fault Detection Method under Variable Speed Conditions," Mechanical Systems and Signal Processing, Vol. 117, pp. 347-360, 2019

14. C. Yang and M. P. Jia, "A Novel Weak Fault Signal Detection Approach for a Rolling Bearing using Variational Mode Decomposition and Phase Space Parallel Factor Analysis," Measurement Science and Technology, Vol. 30, No. 11, pp. 115004, 2019

15. Z. Yuan, L. Zhang, and L. Duan, "A Novel Fusion Diagnosis Method for Rotor System Fault based on Deep Learning and Multi-Sourced Heterogeneous Monitoring data,” Measurement Science and Technology, Vol. 29, No. 11, pp. 115005, 2018

16. B. Guo, Y. Xu, Y. Xia, G. Wilson, and S. Y. Liu, "An Intelligent Time-Adaptive Data-Driven Method for Sensor Fault Diagnosis in Induction Motor Drive System," IEEE Transactions on Industrial Electronics, Vol. 66, No. 12, pp. 9817-9827, 2019

17. Z. Li, J. Y. Li, Y. Wang, and K. S. Wang, "A Deep Learning Approach for Anomaly Detection based on SAE and LSTM in Mechanical Equipment," International Journal of Advanced Manufacturing Technology, Vol. 103, No. 1-4, pp. 499-510, 2019

18. K. Elangovan, Y. K. Tamilelvam, R. E. Mohan, M. Iwase, T. Nemoto, and K. L. Wood, "Fault Diagnosis of a Reconfigurable Crawling-Rolling Robot based on Support Vector Machines," Applied Sciences, Vol. 7, No. 10, pp. 1025, 2017

19. P. Santos, L. Villa, A. Reñones, A. Bustillo, and J. Maudes, "An SVM-based Solution for Fault Detection in Wind Turbines," Sensors, Vol. 15, No. 3, pp. 5627-5648, 2015

20. C. Li, R. V. Sanchez, G. Zurita, M. Cerrada, D. Cabrera, and R. E. Vasquez, "Multimodal Deep Support Vector Classification with Homologous Features and its Application to Gearbox Fault Diagnosis," Neurocomputing, Vol. 168, pp. 119-127, 2015

21. Y. Zhou and R. Cao, "The Artificial Neural Network Prediction Algorithm Research of Rail-Gun Current and Armature Speed based on B-Dot Probes Array," Measurement, Vol. 133, pp. 47-55, 2019

22. D. P. Mandic and J. Chambers, "Recurrent Neural Networks for Prediction: Learning Algorithms, Architectures and Stability," John Wiley and Sons, Inc., 2001

23. H. Jaeger, "The 'Echo State' Approach to Analyzing and Training Recurrent Neural Networks-with an Erratum Note," German National Research Center for Information Technology GMD Technical Report, Bonn, Germany, Vol. 148, No. 34, pp. 13, 2001

24. W. Maass, T. Natschläger, and H. Markram, "Real-Time Computing Without Stable States: A New Framework for Neural Computation based on Perturbations," Neural Computation, Vol. 14, No. 11, pp. 2531-2560, 2002

25. D. Verstraeten, B. Schrauwen, M. D'Haene, and D. Stroobandt, "An Experimental Unification of Reservoir Computing Methods," Neural Networks, Vol. 20, No. 3, pp. 391-403, 2007

26. D. R. Cabrera, F. Sancho, M. Cerrada, R. V. Sanchez, and F. Tobar, "Echo State Network and Variational Autoencoder for Efficient One-Class Learning on Dynamical Systems," Journal of Intelligent and Fuzzy Systems, Vol. 34, No. 2, pp. 1-11, 2018

27. Z. X. Zheng, S. Morando, M. C. Marion-Pera, D. Hissel, L. Larger, and R. Martinenghi, "Brain-Inspired Computational Paradigm Dedicated to Fault Diagnosis of PEM Fuel Cell Stack," International Journal of Hydrogen Energy, Vol. 42, No. 8, pp. 5410-5425, 2017

28. K. He, Z. J. Yang, Y. Bai, J. Y. Long, and C. Li, "Intelligent Fault Diagnosis of Delta 3D Printers using Attitude Sensors based on Support Vector Machines," Sensors, Vol. 18, No. 4, pp. 1298, 2018 